\title{
MV Power Grids Integration of a Resistive Fault Current Limiter Based on HTS-CCs
}

\author{
D. Colangelo and B. Dutoit
}

\begin{abstract}
Due to the energy demand growth and distributed generation (DG) units penetration, a substantial increase of the rated short-circuit current of the electrical lines is expected. As a consequence, the electrical grid infrastructure needs to be extended or drastically renovated. In this context, resistive superconducting fault current limiters (RFCLs) based on high temperature superconducting coated conductors (HTS-CCs) represent a promising technology to limit the upgrading costs. Thanks to recent improvements on HTS-CCs performances, RFCLs are now close to commercial applications. However, as they are novel devices, their real impact on the electricity network remains an open issue. In particular, the subject of this research is to study the grid integration of the RFCL designed within the European project ECCOFLOW [1]. The device has been simulated in two applications in two different typologies of existing medium voltage grids: RFCL used as busbars coupler and RFCL used as transformer feeder. This contribution is the continuation of previous works [2], [3], where the effects of symmetrical and unsymmetrical short-circuits on inhomogeneous HTS-CCs have been extensively analyzed.
\end{abstract}

Index Terms-Power Network Modelling, Superconducting Fault Current Limiters, Coated Conductors, MV grids.

\section{INTRODUCTION}

$\mathbf{F}$ OR multiple reasons (DG units penetrations, increase of power demand etc..), the rated short circuit of MV distribution lines is in constant increase. Medium voltage power grids need to be scaled to higher short circuit current. It is well known [4], [5] that RFCLs can allow to couple separate sub-grids doubling the short-circuit power while effectively limiting the current when a fault occurs. In the recent years several project focus on RFCLs [6]-[8]. In particular, ECCOFLOW is the first one where the same RFCL is designed for two different applications: busbar coupler and transformer feeder. This stage of the project requires to study the device integration in the grids where it will operate. The grid typology (voltage level, line impedance etc...) has a deep influence on the limiting performance of RFCLs. For instance, a fault current slightly higher than the operating current of the line (e.g. single or double phases short-circuits) may be ignored by the conventional protections but may have disruptive effects on the limiter due to critical current $\left(I_{c}\right)$ inhomogeneity [9]. The number of paralleled tapes $n_{H T S-C C}$ that composes the

Manuscript received 9 October 2012.

Corresponding author's e-mail: daniele.colangelo@epfl.ch.

D.Colangelo and B. Dutoit are with École polytechnique fédérale de Lausanne, EPFL-SCI-IC-BD , Station 14, 1015 Lausanne, Switzerland.

The research leading to these results has received funding from the European Union Seventh Framework Programme (FP7/2007 - 2013) under grant agreement No. 241285

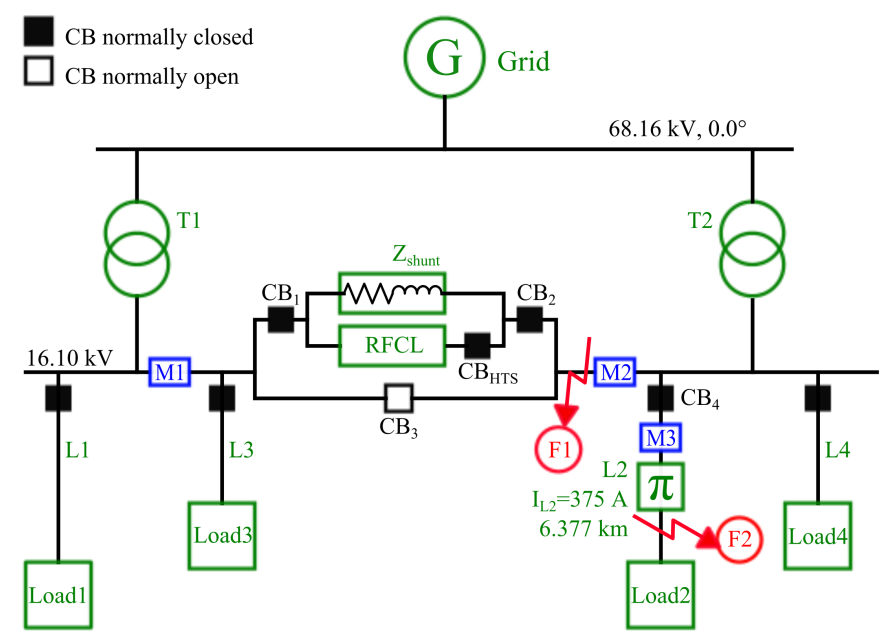

Fig. 1. Single line diagram of the busbar coupling application of the RFCL prototype. The diagram shows the short-circuit locations (F1 and F2) and the measurement points (M1, M2 and M3).

RFCL module is related to the nominal current of the line $\left(I_{\text {line }}\right)$ where the device operates by equation

$$
n_{H T S-C C}=\left(\frac{\sqrt{2} I_{\text {line }}}{I_{c, \text { min }}}\right) \cdot k
$$

where $I_{c, \min }$ is the minimum critical current of the tapes and $k$ is a security factor, generally 1.2 . In practice, the RFCL module limits the fault during the transient phase of the short circuit, hence, the HTS-CCs can be designed to withstand the current for few electrical cycles. Once the transient is elapsed, the fault current is totally diverted on an external shunt paralleled to the device. The fault current can be described by the following expression,

$$
\begin{gathered}
i_{F}(t)=\frac{\frac{U_{0}}{\sqrt{3}}}{\left|Z_{\text {fault }}\right|}(\underbrace{\sin (\omega t+\alpha-\varphi)}_{\text {steady-state }}-\underbrace{e^{-\frac{t}{\tau}} \sin (\alpha-\varphi)}_{\text {transient }}) \\
Z_{\text {fault }}=\left(R_{g}+R F\right)+j \omega L_{g}
\end{gathered}
$$

where $\varphi$ and $\tau$ are respectively the electrical angle and the time constant of the circuit, $R_{g}$ and $L_{g}$ are respectively the resistance and the inductance of the grid "seen" from the fault location. The fault angle $\alpha$ has been chosen in order to maximize the transient term of equation 2. As the MV grids are in general strongly inductive $\left(\varphi \simeq \frac{\pi}{2}\right), i_{F}$ assumes its maximum value when $\alpha$ is circa $2 n \pi$ with $n \in \mathbb{Z}$. In time domain, it corresponds to simulate the fault around the integer 


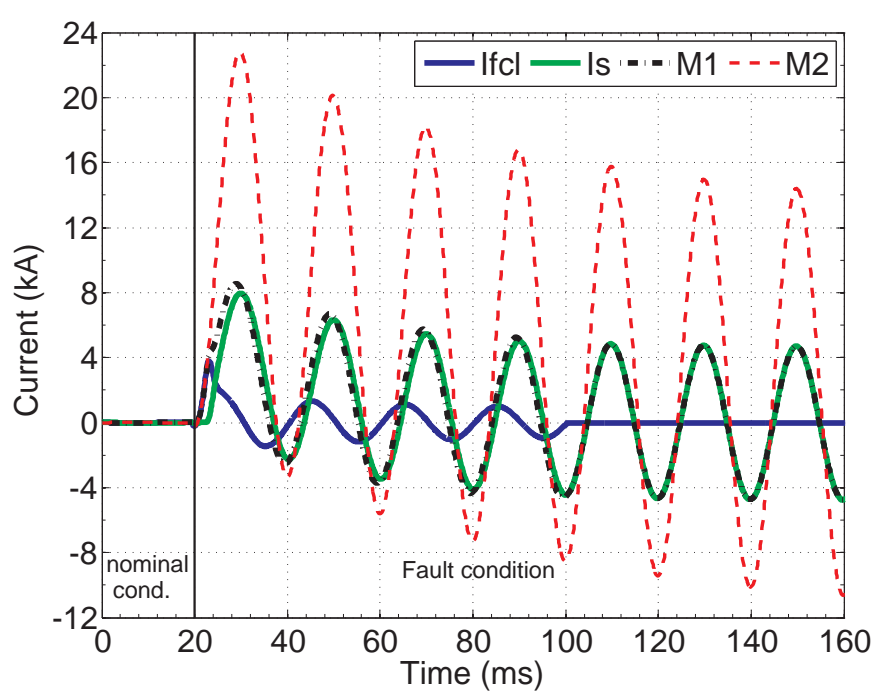

Fig. 2. Device limiting performance under three phases short-circuit in the busbar coupling application. Current sharing between the external shunt $\left(I_{s}\right)$ and the RFCL module $\left(I_{f c l}\right)$ when the fault is located in point F1.

multiples of the electrical period $T$. This manuscript reports the preliminary simulations of the ECCOFLOW prototype in operating conditions. For both applications, busbar coupler and transformer feeder, the device respond against faults of different intensity (symmetrical and unsymmetrical faults) and location (e.g. busbar line and downstream transmission line) has been analyzed. Symmetrical and unsymmetrical faults have been simulated inserting fictitious resistance $(R F)$ in the fault location. The model of the RFCL has been developed in SimPowerSystem ${ }^{\mathrm{TM}}$ and is reported in details in previous works [2], [3]. The parameters of the limiter are reported in table I.

TABLE I

FAULT CURRENT LIMITER PARAMETERS SUMMARY

\begin{tabular}{|l|c|c|}
\hline Parameter & Expression & Description \\
\hline$U_{0}$ & $24 \mathrm{kV}$ (phase-to-phase) & Nominal voltage \\
$I_{n, R M S}$ & $1 \mathrm{kA}$ & Nominal current \\
$I_{c}$ & $1.9 \pm 0.2 \mathrm{kA}(5 / /$ tapes) & Total $I_{C}$ \\
$Z_{\text {shunt }}$ & $1.4 \Omega$ & External shunt impedance \\
$t_{C B_{H T S}}$ & $80 \mathrm{~ms}$ & $C B_{H T S}$ tripping time \\
$t_{\text {fault }}$ & $1 \mathrm{~s}$ & Maximum fault time \\
$L_{\text {parelled }}$ & $180 \mathrm{~m}$ & Length single tape per phase \\
$T_{\text {max }}$ & $360 \mathrm{~K}$ & max temperature allowed \\
\hline
\end{tabular}

\section{BUSBAR COUPLING APPLICATION}

The RFCL has to be integrated into a portion of the MV network of the Balearic island of Mallorca operated by the Spanish utility company Endesa [10]. As shown in the single line diagram of Fig. 1, faults have been simulated in point F1 and F2. In the worst case, the device is subjected to a voltage drop of $16 \mathrm{kV}$. Under operating conditions, when the grid is balanced, just a few amps flows on the busbar line.

\section{A. Fault location F1}

When a fault occurs, in F1 the RFCL can guarantee only a partial protection. Indeed, the device is able to limit the

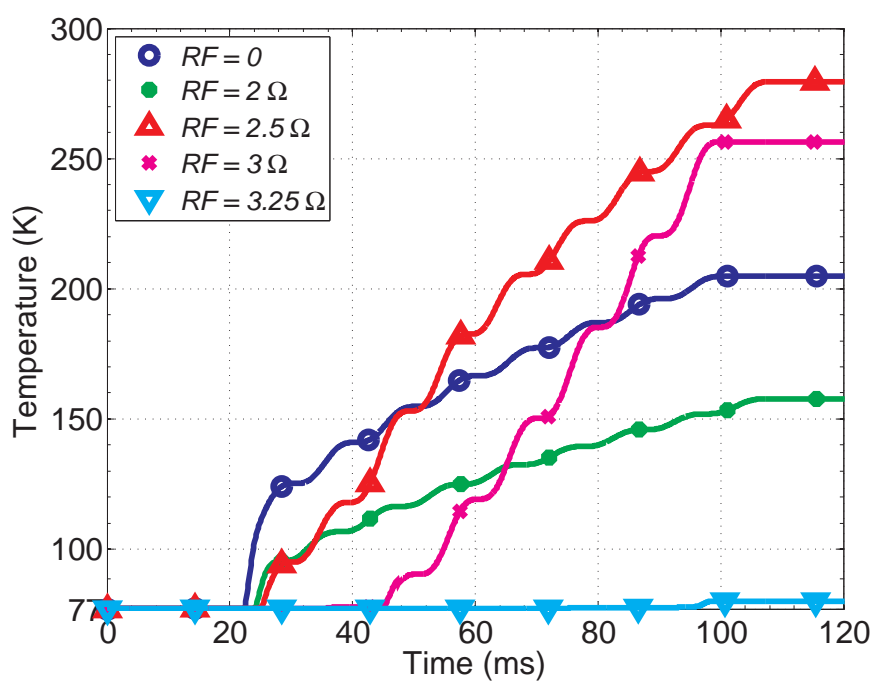

Fig. 3. Temperature profiles for different values of $R F$ in the busbar coupler application with fault location F1. For $R F>4 \Omega$ the current through RFCL is lower than $I_{c, \text { min }}$ (inset).

current from transformer $\mathrm{T} 1$ but the one through $\mathrm{T} 2$ will directly feed the fault. Under a clear three phases short-circuit, the current peak is $23 \mathrm{kA}$ in $\mathrm{M} 1$ and $8 \mathrm{kA}$ in M2 (Fig. 2). Therefore, the maximum prospective short-circuit current $\left(I_{p s c c}\right)$ can be estimated roughly around $32 \mathrm{kA}$. From a thermal point a view, $I_{p s c c}$ does not represent a problem. The high current leads the HTS-CCs to homogeneous quench. With a proper design of superconductor length, it is relatively easy to face the $T_{\max }$ criterion. As expected, lowering the fault current ( $R F$ increase) underlines the inhomogeneity (in terms of $I_{c}$ ) problem. In particular, our simulations estimate that the maximum temperature of the device is reached for $R F=$ $2.5 \Omega$ (red line Fig. 3). However, the most dangerous case is when $R F \simeq 4 \Omega$. The current peak of the limiter $\left(I_{f c l . F 1, p}\right)$ is around $I_{c, \min }$ (inset Fig. 5). Due to the inhomogeneity, only a few parts (with lower $I_{c}$ ) of the HTS-CCs could quench with extremely low dynamics leading to hot spots. In order to avoid thermal take-off, it is necessary to disconnect the device triggering $C B_{H T S}$ within $80 \mathrm{~ms}$.

\section{B. Fault location $F 2$}

This section analyzes the short-circuit downstream line 2 (fault location F2, Fig. 1). The fault is now fed by the two transformers (T1 and T2). According to our simulation, the current peak $\left(I_{L 2 . F 2, p}\right)$ varies between 3 and 11 times the operating current of the line $\left(I_{L 2}\right.$, Fig. 4). With such values, the extinguishment of the fault is guaranteed by the line breakers (e.g. $C B_{4}$ Fig. 1). They are calibrated (tripping current) on the operating line currents and their intervention time is inversely proportional to the entity of the fault. The current on the busbar line assumes low values (inset Fig. 4). Unless the line circuit breakers are not able to trip within $t_{C B_{H T S}}$, the RFCL module does not react to the faults located in F2. However, if $C B_{4}$ is not able to extinguish the fault within $80 \mathrm{~ms}$, the limiter will be disconnected by $C B_{H T S}$ leaving the busbar without protection. The coordination between line circuit breaks and 


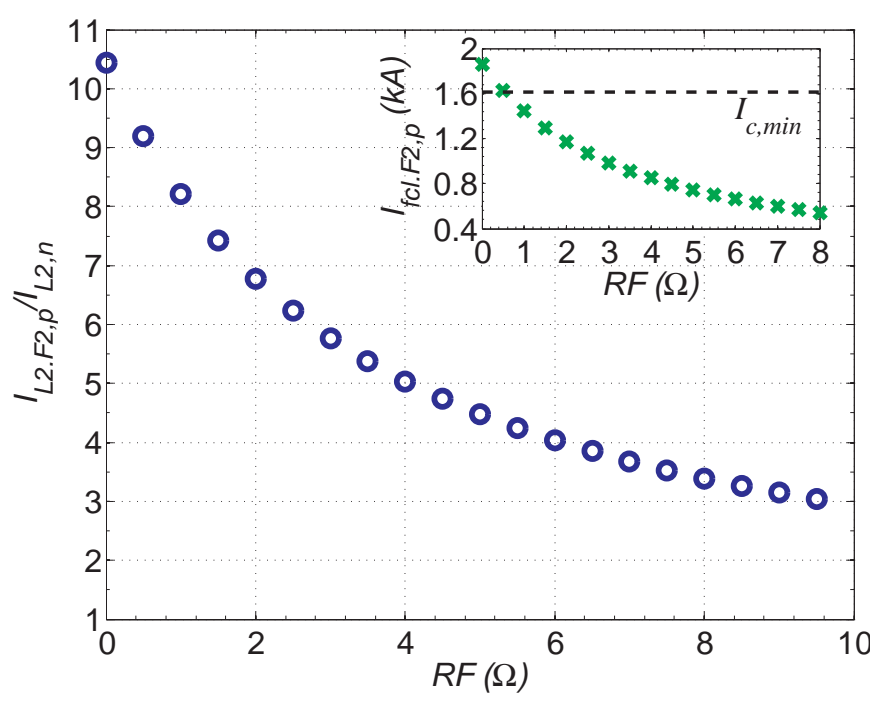

Fig. 4. Ratio between the fault and the nominal current peaks of the short circuited line L2. The inset shows the peak of the current through the device.

$C B_{H T S}$ has to avoid a lack of protection against simultaneous faults.

\section{Following current}

This section treats the busbar following current $\left(I_{f o l}\right) 300$ $\mathrm{ms}$ after the fault is detected. Due to the "partial" protection the RFCL can guarantee, it is possible to distinguish between $I_{f o l}$ measured in $M 1\left(I_{f o l . M 1}\right)$ and the one measured in $M 2$ $\left(I_{f o l . M 2}\right)$. However, only $I_{f o l . M 1}$ flows through the device and it is treated in the section below. As shown in Fig. 5, the maximum long-run current is around $4 \mathrm{kA}$. For $R F$ between $2.5 \Omega$ and $4 \Omega$, the first peak of the fault current is able to trig the quench of the HTS-CCs $\left(I_{f c l . F 1, p}>I_{c, \text { min }}\right.$, inset Fig. 5) whereas, $I_{f o l} 300 \mathrm{~ms}$ is lower than $I_{n, R M S}$. When $R F$ is greater than $4 \Omega$, there is no effect on the limiter

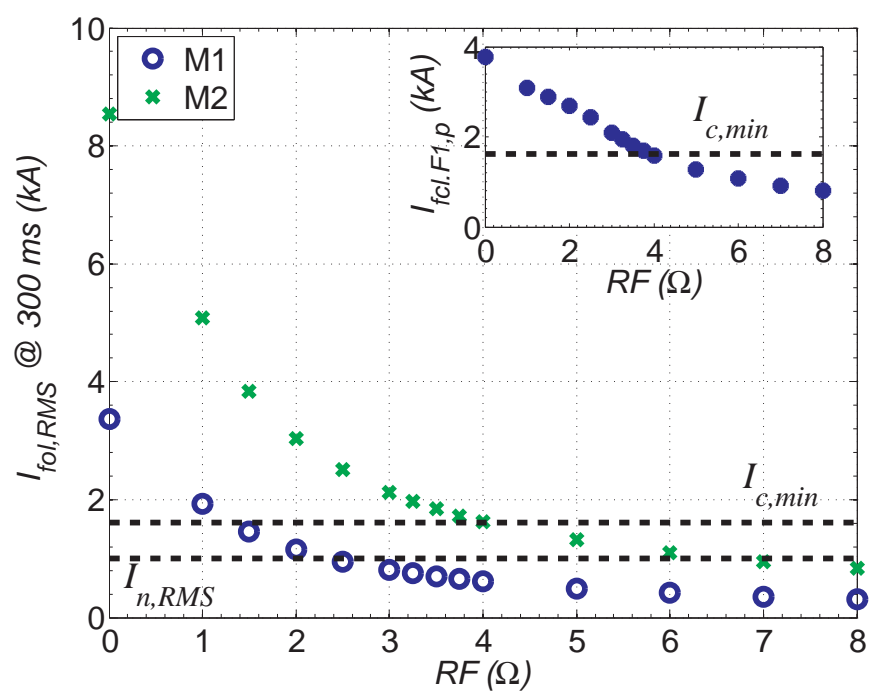

Fig. 5. Following current for different values of $R F$ in the busbar coupling application with fault in F1. The current has been measured $300 \mathrm{~ms}$ after from the fault detection.

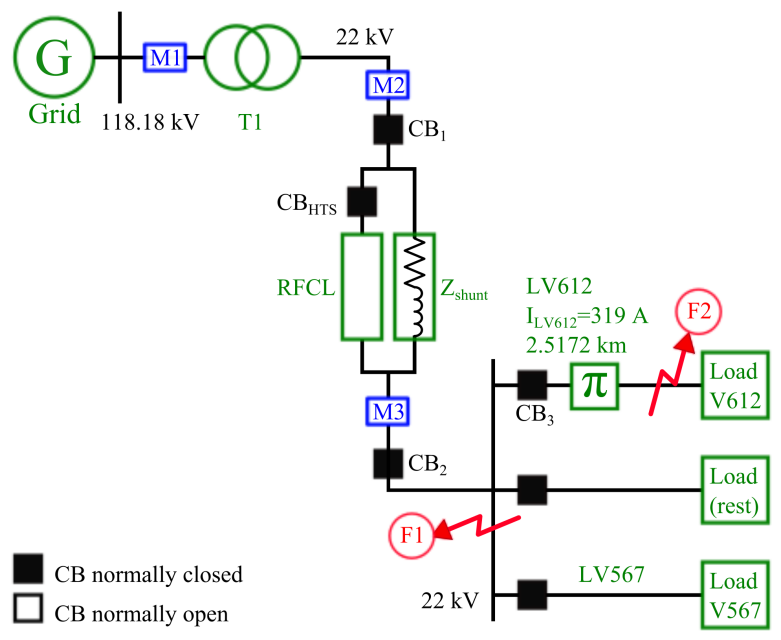

Fig. 6. Single line diagram of the transformer feeder application of the RFCL prototype. The diagram shows the locations of the short-circuits (F1 and F2) and the measurement points (M1, M2 and M3).

$\left(I_{f c l}<I_{c, \min }\right.$, inset Fig. 5). The overcurrent regime has to be extinguished by the convectional circuit breakers. The following current for faults located in F2 have not been considered. As mentioned in section II-B, the proper intervention of the standard protections has to assure the disconnection of line (extinction of the fault condition on the busbar line).

\section{TRANSFORMER FEEDER APPLICATION}

The RFCL model has to be integrated as transformer feeder into a portion of the Slovak electricity network operated by VSE [11]. The diagram of Fig. 6 shows that the device operate now at to $22 \mathrm{kV}$ instead of $16 \mathrm{kV}$ of the previous case. The distribution lines are fed by a single transformer (T1). As the device is installed in series to $\mathrm{T} 1$, the operating current of the line (the current absorbed by the loads) flows through it.

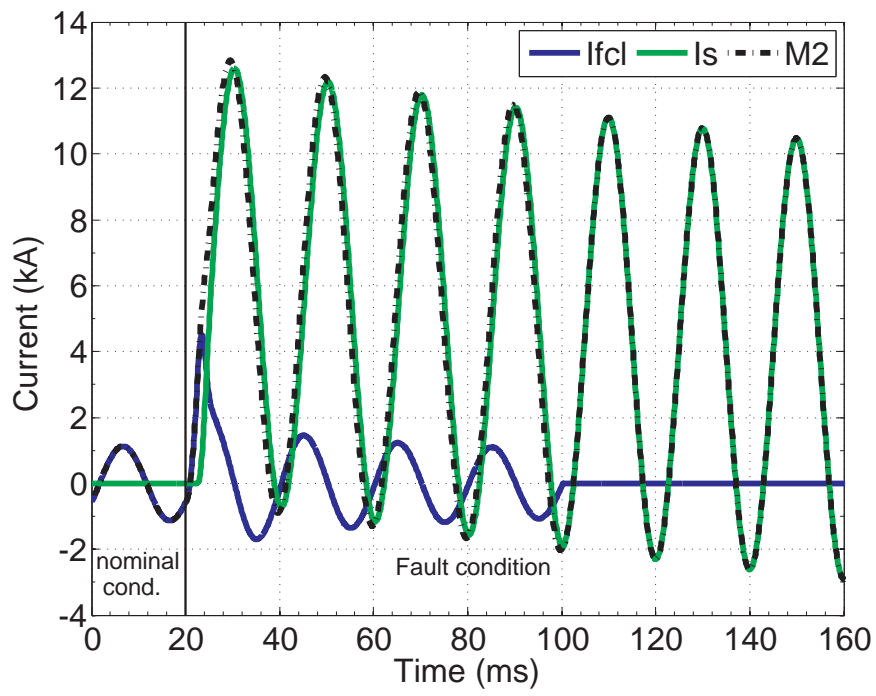

Fig. 7. Current sharing between the external shunt $\left(I_{s}\right)$ and the RFCL module $\left(I_{f c l}\right)$ under three phases short-circuit (fault point $\mathrm{F} 1$ ) in the transformer feeder application. 


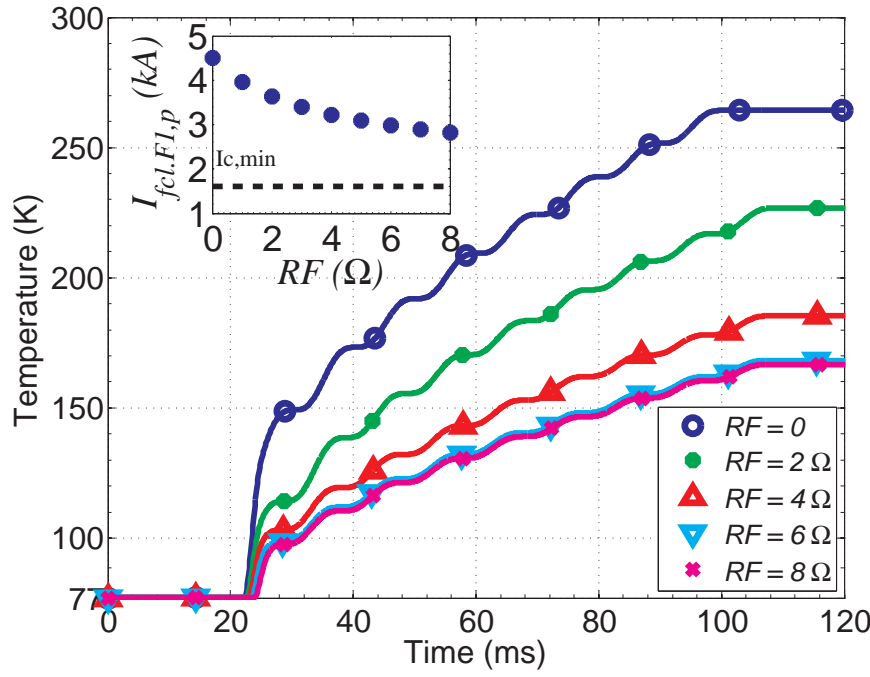

Fig. 8. Temperature profiles for different values of $R F$ in the transformer feeder application with fault location F1. The current through the device is always higher than $I_{c, \min }$ (inset).

\section{A. Fault location F1}

In this application, the RFCL gives a full protection of the line. The first peak of $I_{p s c c}$ is around $13 \mathrm{kA}$ (Fig. 7). Within the whole $R F$ range considered, the high value of the current induces an homogenous quench (see inset Fig. 8), the temperature increase of the device is inversely proportional to $R F$ (Fig. 8). The thermal stability of the RFCL is guaranteed for all analyzed faults.

\section{B. Fault location $F 2$}

The fault current peak $\left(I_{V 612 . F 2, p}\right)$ varies between 4 and 22 times the operating current of the line $I_{V 612, p}$ (Fig. 6). For the same considerations made in subsection II-B, the line circuit breakers will react according to their time-current curve. In

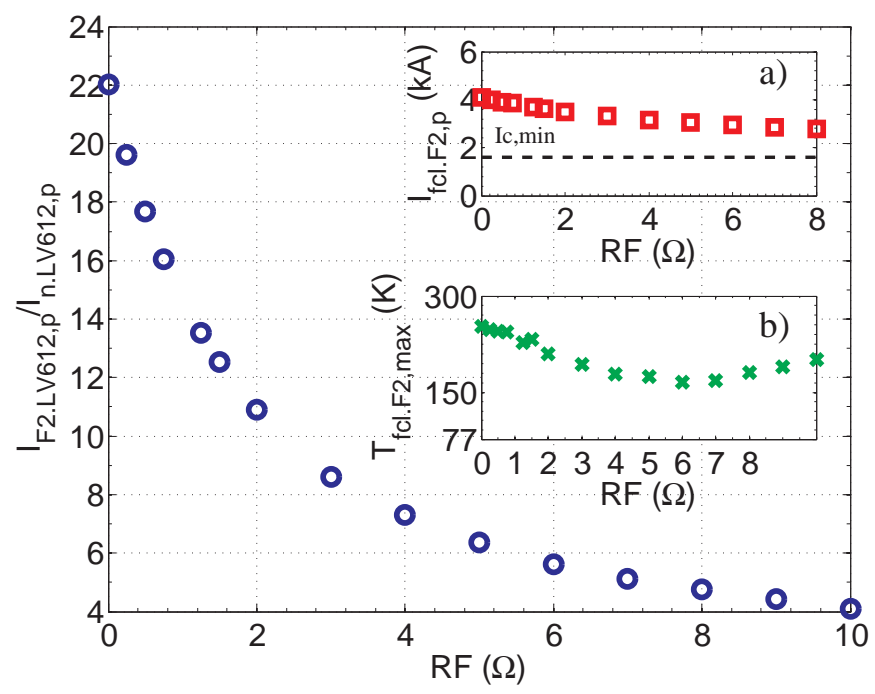

Fig. 9. Ratio between the fault peak and the nominal current of the short circuited line LV612. The inset shows the maximum temperature reached by the device after the transient limitation ( $80 \mathrm{~ms}$ from fault detection).

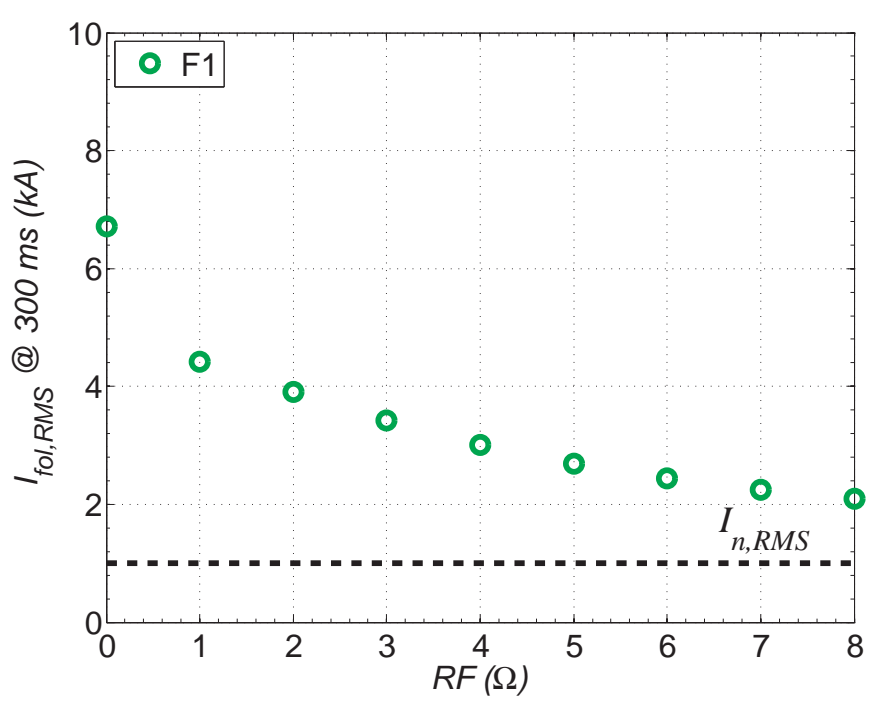

Fig. 10. Following current for different values of $R F$ in the transformer feeder application with fault in F1. The current has been measured after 300 ms from the fault detection.

comparison with the previous case, now the current through the device $\left(I_{f c l . F 2}\right)$ is much higher than $I_{c, \min }$ for all $R F$ values (Fig. 9 inset $a$ ). To avoid thermal instability, $C B_{H T S}$ has to trip within $t_{C B_{H T S}}$ diverting the current on the external shunt. If the fault regime persists longer than $t_{f a u l t}$, all the lines will be disconnected ( $C B 1$ and $C B 2$ trip). Therefore, even though a fault located in F2 is not dangerous for the thermal stability of the device (Fig. 9 inset $b$ ), it could generate an outage on the healthy lines not directly affected by the short-circuit.

\section{Following current}

Given that the electrical potential difference applied on the device is noticeably higher than in the previous case, using an external shunt with the same impedance $\left(Z_{\text {shunt }}\right)$ as in the busbar case implies a higher current after the transient limitation of the fault, Fig. 10. Anyway, the increase of $I_{f o l}$ is still in an acceptable range and it does not represent a problem for the utility company that will host the device.

\section{CONCLUSiON}

The RFCL designed within the ECCOFLOW project is close to the field test. Therefore it is fundamental to simulate its behavior before being inserted in the test grids. In this respect, the RFCL model has been interfaced with the line parameters of the two MV grids where it will operates, respectively as busbar coupler and transformer feeder. Our simulations foresee the impact of the device against faults of different intensity and locations. They prove that even though the device will react to faults with different performances, the same device can be safely integrated in various MV networks with different characteristics. Moreover, the simulation are a solid base to study the coordination between the RFCL and the existing protections of the system. 


\section{REFERENCES}

[1] Eccoflow website [Online]. http://www.eccoflow.org.

[2] D. Colangelo et al., "Impact of Inhomogeneities in HTS Coated Conductors for Resistive FCLs", Proceedings of EUCAS 2011, The Hague, Netherlands, September 18-23, 2011.

[3] D. Colangelo and B. Dutoit, "Inhomogeneity Effects in HTS Coated Conductors Used as Resistive FCLs in Medium Voltage Grids", Supercond. Sci. Tech., vol. 25, 2012, 095005.

[4] C. Neumann, "Superconducting Fault Current Limiter (SFCL) in the Medium and High Voltage Grid", IEEE Power Engineering Society General Meeting, Montreal, Canada, 2006, art. no. 1709336.

[5] M. Noe and M. Steurer, "High-temperature superconductor fault current limiters: concepts, applications, and development status", Supercond. Sci. Tech., vol. 20, pp. R15-R29, March 2007.

[6] J. Bock et al., "CURL 10: Development and Field-Test of a $10 \mathrm{kV} / 10$ MVA Resistive Current Limiter Based on Bulk MCP-BSCCO 2212", IEEE Trans. Appl. Supercond., vol. 15, no. 2, pp. 1955-1960, 2005.

[7] S. Elschner et al: "ENSYSTROB-Resistive Fault Current Limiter Based on Coated Conductors for Medium Voltage Application", IEEE Trans. Appl. Supercond., vol. 21,no. 3, pp. 12091212, 2011.

[8] M. Noe et al., "Conceptual Design of a 24 kV, 1 kA Resistive Superconducting Fault Current Limiter", IEEE Trans. Appl. Supercond., vol. 22, no. 3, 2012.

[9] P. Tixador and N. T. Nguyen, "Design of ReBaCuO-coated conductors for FCL", Supercond. Sci. Tech., vol. 25, 2012, 014009.

[10] Endesa website [Online] Available: http://www.endesa.com

[11] VSE website [Online] Available: http://www.vse.sk 